\title{
Identifying potential therapeutic targets of Tang-Yi-Ping for the treatment of impaired glucose tolerance: a tandem mass tag- labeled quantitative proteomic analysis
}

\author{
Jie $\mathrm{Li}^{1,2}$, Shuai $\mathrm{Bu}^{3}$, Honglei $\mathrm{Zhou}^{4}$, Siling $\mathrm{Bi}^{3}$, Yunsheng $\mathrm{Xu}^{2}$ \\ ${ }^{1}$ College of the Second Clinical Medicine, Shandong University of Traditional Chinese Medicine, Jinan, China; ${ }^{2}$ Department of Endocrinology \\ Medicine, the Second Affiliated Hospital of Shandong University of Traditional Chinese Medicine, Jinan, China; ${ }^{3}$ Department of Cardiovascular \\ Medicine, the Second Affiliated Hospital of Shandong University of Traditional Chinese Medicine, Jinan, China; ${ }^{4}$ College of pharmacy, Shandong \\ University of Traditional Chinese Medicine, Jinan, China \\ Contributions: (I) conception and design: J Li; (II) administrative support: Y Xu; (III) provision of study materials or patients: J Li, Y Xu; (IV) \\ collection and assembly of data: S Bu, H Zhou; (V) data analysis and interpretation: S Bi, Y Xu; (VI) manuscript writing: All authors; (VII) final \\ approval of manuscript: All authors. \\ Correspondence to: Yunsheng Xu. Department of Endocrinology Medicine, the Second Affiliated Hospital of Shandong University of Traditional \\ Chinese Medicine, Jinan 250001, China. Email: xysnfm65@163.com.
}

Background: This study uses the tandem mass tag (TMT)-labeled quantitative proteomic analysis to identify potential therapeutic protein targets of a Chinese prescription called Tang-Yi-Ping (TYP) for the treatment of impaired glucose tolerance (IGT) in rats.

Methods: A total of 31 specific-pathogen free (SPF) male Wistar rats were used in our study. Ten were randomly selected as a control group, while 21 received a high-sugar and high-fat diet combined with an intraperitoneal injection of streptozotocin to establish IGT subjects. After eliminating 2 rats without successful modeling, 19 were randomly divided into a TYP group (n=9) and IGT model group $(\mathrm{n}=10)$. The TYP group was given a TYP decoction of $6.36 \mathrm{mg} / \mathrm{kg}-1 / \mathrm{d}-1$. After 8 weeks of intervention, blood glucose-related indicators were measured, and cell morphology was observed by hematoxylin and eosin (HE) staining. TMT-labeled proteomic analysis was applied to detect the differentially expressed proteins (DEPs) in the pancreases of the three groups. The intersection of the DEPs in both the TYP group and IGT model group underwent Gene Ontology (GO) and Kyoto Encyclopedia of Genes and Genomes (KEGG) pathway analyses to identify the related biological functions and signal transduction pathways. Finally, western blot $(\mathrm{WB})$ was used to verify the TMT proteomics results.

Results: TYP can effectively reduce blood glucose and improve islet morphology in IGT rats. We identified a total of 16 potential therapeutic protein targets of TYP, 4 of which were upregulated, while 12 were downregulated, including Rbp4, Fam3b, Flot2, etc. [fold change $(\mathrm{FC})>1.1, \mathrm{P}<0.05$ ]. The significant signal transduction pathways included arginine and proline metabolism, glyceride metabolism, glycerophospholipid metabolism, mTOR, Wnt, and insulin signaling pathways.

Conclusions: For anti-IGT therapy, we found TYP regulates 16 protein targets, multiple biological functions, and multiple signal transduction pathways. This study thus makes a significant contribution to identifying new potential therapeutic targets for treating IGT.

Keywords: Impaired glucose tolerance (IGT); Tang-Yi-Ping (TYP); tandem mass tag proteomics (TMT proteomics); protein targets

Submitted Aug 05, 2021. Accepted for publication Oct 09, 2021.

doi: 10.21037/atm-21-4257

View this article at: https://dx.doi.org/10.21037/atm-21-4257 


\section{Introduction}

The term "pre-diabetes" is used to describe a condition that involves impaired glucose tolerance (IGT) or impaired fasting glucose (IFG). IGT and IFG are intermediate states in glucose metabolism that exist between normal glucose tolerance and overt diabetes (1). Studies have shown that people with pre-diabetes tend to develop type 2 diabetes mellitus (T2DM) within 10 years of its diagnosis. Even before developing diabetes, these people also have an increased risk of cardiovascular disease and death. In the $9^{\text {th }}$ edition of the International Diabetes Federation (IDF) Diabetes Atlas [2019], it is estimated that worldwide there are 373.9 million adults aged $20-79$ years $(7.5 \%$ of the adult population) who have IGT. In China, there are approximately 54.5 million adults with IGT (2). At present, it is recognized by the medical community as a necessary stage in the pathogenesis of T2DM, and also the only stage of T2DM that can be controlled and reversed (3). For this reason, taking appropriate measures to prevent IGT from developing into T2DM can reduce the burden of medical treatment, which is of great significance to both patients and society. The etiology and pathogenesis of IGT are yet to be fully elucidated, and insulin resistance (IR) and functional deficiency of pancreatic $\beta$-cells are the primary pathological basis of IGT (4). IGT is the earliest manifestation of a glucose metabolism disorder and involves the secretion of insulin after meals, and is often accompanied by other protein and fat-metabolism disorders. Currently, there are very few drugs used for treating IGT, the main ones being metformin and acarbose. However, these treatments have certain limitations due to side effects, such as hepatorenal toxicity, gastrointestinal reactions, and so on (5-7). Therefore, the medical community is now actively searching for more complementary and alternative drugs that are safe, effective, and have little side effects. A series of studies have confirmed that the Chinese prescription, Tang-Yi-Ping (TYP), which is made from Astragalusmembranaceus, Rhizoma Dioscoreae, Atractylodes Lancea, Radix Bupleuri, Radix Paeoniae Alba, Rhizoma Coptidis, Eupatorium fortune Turcz, Radix Scrophulariae, Puerarialobata, and Ramulus Euonymi, can treat IGT effectively and safely, improve the proliferation of pancreatic $\beta$-cells, and protect the number and function of $\beta$-cells. Previous studies have shown that TYP's mechanism relates to the downregulation of the thioredoxin interacting protein (TXNIP), and the promotion of the expression of the musculoaponeurotic fibrosarcoma oncogene homolog A $(\mathrm{MaFA})$ gene $(8,9)$. Owing to the multiple components and targets characteristic of traditional Chinese medicine for treating diseases, it is necessary to use tandem mass tag (TMT)-labeled quantitative proteomic technology to identify all the protein targets of TYP in treating IGT. Elucidating the potential targets and signaling pathways of this prescription can thus provide a basis for finding new potential therapeutic targets for treating IGT, which holds great research value.

This study is the first report to use TMT-labeled quantitative proteomics technology and bioinformatics to identify the protein targets, biological processes (BPs), and related signaling pathways involved in the treatment of IGT model rats with TYP. The workflow of this study is shown in Figure 1. We present the following article in accordance with the ARRIVE reporting checklist (available at https:// dx.doi.org/10.21037/atm-21-4257).

\section{Methods}

\section{Model preparation and grouping}

All animal experiments protocol were performed strictly in compliance with Chinese guidelines, including the Standards for Laboratory Animals (GB14925-2001) and the Guideline on the Humane Treatment of Laboratory Animals (MOST 2006a), and all animal procedures were approved by the Animal Ethics Committee of Shandong University of Traditional Chinese Medicine (No. SDUTCM20190520001). A protocol was prepared before the study without registration. Thirty-one specificpathogen free (SPF) male Wistar rats were purchased from Jinan Pengyue Biological Breeding Co. Ltd. (No. 1107261911005606 , China). They were 5 weeks old, weighed130 g, and were raised and observed in the barrier system of the Animal Experimental Center of Shandong University of Traditional Chinese Medicine at a room temperature of $21 \pm 2{ }^{\circ} \mathrm{C}$ (humidity $50-70 \%$ ), with regular day and night hours (12-12 h). All rats were adaptively fed for one week. Our study began by randomly selecting 10 rats as the control group, while 21 were fed a high-sugar and high-fat diet (diet formula: $67 \%$ rat maintenance diet + $10.0 \%$ lard $+20.0 \%$ sucrose $+2.5 \%$ sodium cholate $+2.5 \%$ cholesterol) for 4 weeks. After overnight fasting, they were weighed and given a one-time intraperitoneal injection of $15 \mathrm{mg} / \mathrm{kg}$ streptozotocin (\#18883-66-4, Sigma-Aldrich, St. Louis, MO, USA). In line with previous literature $(10,11)$, an oral glucose tolerance test (OGTT) was carried out, and the blood glucose value was measured from the tail vein of 


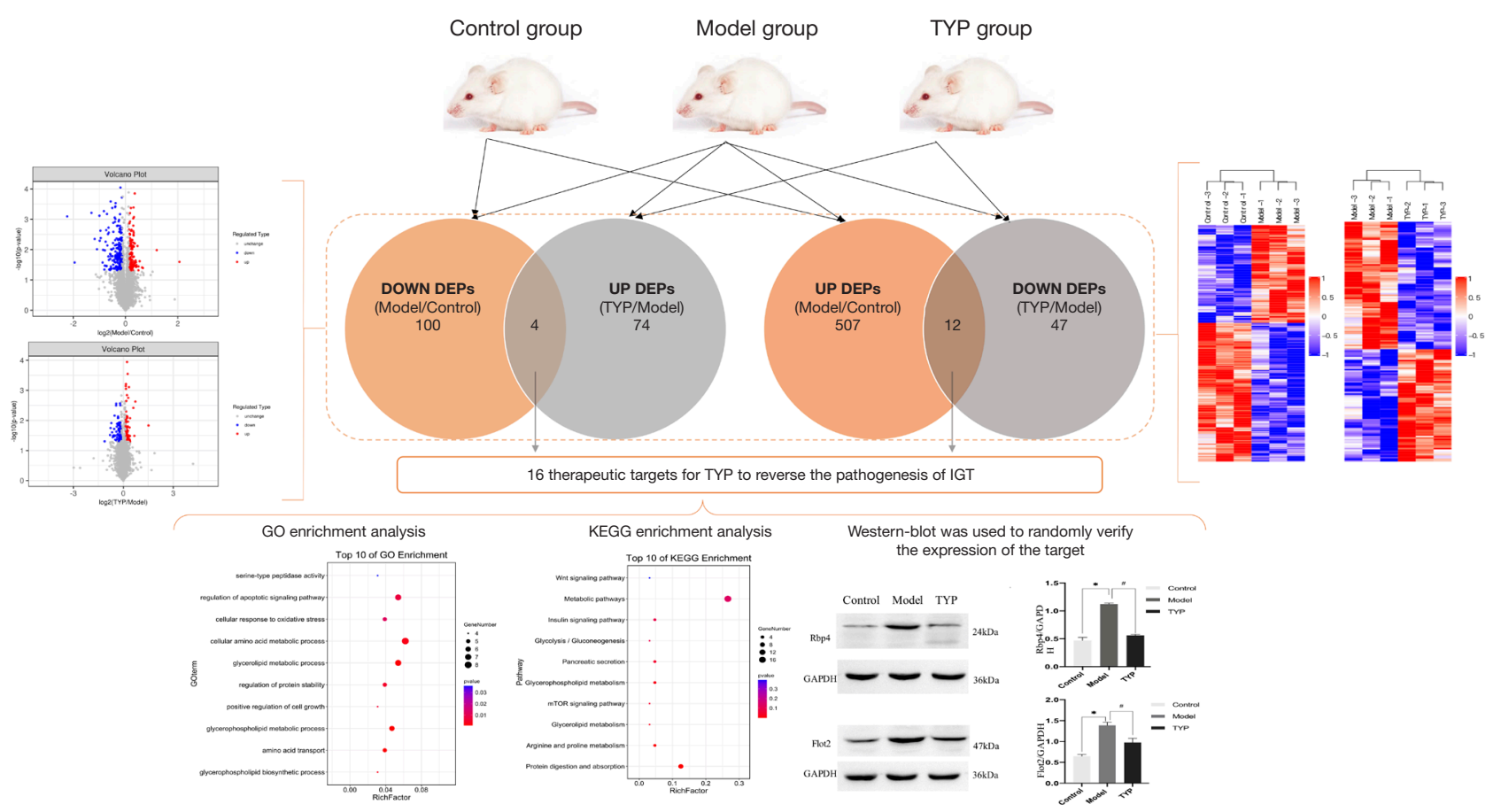

Figure 1 The main flow chart of this study. Rbp4: *, $\mathrm{P}<0.05$, vs. Control; ", $\mathrm{P}<0.05$, vs. IGT. Flot: *, $\mathrm{P}<0.05$, vs. Control; ", $\mathrm{P}<0.05$, vs. IGT. IGT, impaired glucose tolerance.

rats. To be included in the study, IGT rats were required to meet the following OGTT test criteria: fasting blood glucose $(\mathrm{FBG})<7 \mathrm{mmol} / \mathrm{L}$, and 2-hour plasma glucose (2hPG) between 7.8 and $11.1 \mathrm{mmol} / \mathrm{L}$. After removing two unsuccessful modeling rats, the remainder were randomly divided into a TYP group ( $\mathrm{n}=9$ ) and IGT model group $(\mathrm{n}=10)$. TYP is composed of Astragalus membranaceus, Rhizoma Dioscoreae, Atractylodes Lancea, Radix Bupleuri, Radix Paeoniae Alba, Rhizoma Coptidis, Eupatorium fortunei Turcz, Radix Scrophulariae, Pueraria lobata, and Ramulus Euonymi. All these Chinese medicine granules were provided by Jiangyin Tianjiang Pharmaceutical Co., Ltd. (\#19030041; \#19100322; \#19121971; \#19110591; \#19021711; \#19110471; \#1910024; \#19111441; \#19110761; and \#19110971, China). Based on the results of our previous study and pre-experiment, the TYP group was given the Chinese medical decoction $\left(6.36 \mathrm{mg} \cdot \mathrm{kg}^{-1} \cdot \mathrm{d}^{-1}\right)$ by gavage $(3$ $\mathrm{mL}$ each time). Meanwhile, the control and IGT model groups were given the same amount of distilled water by gavage. The body weight and OGTT test results (FBG and $2 \mathrm{hPG}$ ) of each group were measured at a fixed time every week. After 8 weeks, the rats were anesthetized after fasting for 12 hours. The pancreatic tissues were then taken out to detect a series of related-indicators, including hematoxylin $\&$ eosin (HE) staining, TMT-labelled quantitative proteomic analysis, and western blot (WB) detection.

\section{HE staining to observe the morphology and structure of the pancreas in the 3 groups}

An appropriate amount of rat pancreatic tissue was fixed in $10 \%$ formaldehyde solution for 12 hours, dehydrated in ethanol, rendered transparent in xylene, embedded in paraffin, cut into sections with a thickness of $4 \mu \mathrm{m}$, dried at $45{ }^{\circ} \mathrm{C}$, and sealed with HE staining. The morphology of the pancreas and the distribution of islet cells were then observed under microscope.

\section{TMT-labeled quantitative proteomics analysis}

To obtain the difference in protein expressions between the groups, we used TMT-labeled quantitative proteomics technology. This analysis process mainly involved protein extraction, peptide digestion, TMT labeling, chromatographic fractionation, liquid chromatographytandem spectroscopy (LC-MS/MS) data acquisition, 
database searches, GO and KEGG biological enrichment analysis, and other steps.

For our study, we first randomly selected three pancreas samples separately from the three groups. The protein was extracted and quantified for each sample, and the filter aided proteome preparation (FASP) method was used for trypsin digestion, desalting, and peptide quantification. Next, 100 $\mu \mathrm{g}$ of peptide was extracted from each sample, and labeled strictly by the instructions of the TMT labeling kit (Thermo Fisher Scientific Inc., Waltham, MA, USA). The labeled peptides of each group were mixed in equal amounts, and fractionated using the High $\mathrm{pH}$ Reversed-Phase Peptide Fractionation Kit (Thermo Fisher Scientific Inc.). We then performed LC-MS/MS data acquisition. Each sample was separated by an ultra-high performance EASYnLC system with a nanoliter flow rate, and the sample was chromatographed and underwent mass spectrometry analysis by a Q-Exactive mass spectrometer (Thermo Fisher Scientific Inc.). The differentially expressed proteins (DEPs) between the groups were selected with the following criteria: fold change $(\mathrm{FC})>1.1, \mathrm{P}$ value $<0.05$. Hierarchical cluster analysis and subcellular location analysis were also performed on DEPs between (IGT model group vs. control group) and (TYP group $v s$. IGT model group). From these DEPs, we identified protein targets that had an abnormal expression after IGT onset, and normal expression after TYP treatment. Through this, we determined the therapeutic targets of TYP for treating IGT. More specifically, the upregulated DEPs (IGT model group vs. control group) were intersected with the downregulated DEPs (TYP group $v s$. IGT model group), making them the specific targets for upregulated IGT after onset, and normal expression after TYP intervention. Furthermore, the downregulated DEPs (IGT model group vs. control group) were intersected with the upregulated DEPs (TYP group $v s$. IGT model group), making them the specific targets of downregulated IGT after onset, and normal expression after TYP treatment. To conclude the analysis, the intersection of the DEPs in the IGT model group, as well as the DEPs in the TYP group, were taken to perform Gene Ontology (GO) and Kyoto Encyclopedia of Genes and Genomes (KEGG) pathway analyses to identify the related biological functions and signal transduction pathways. Only functional categories and pathways with $\mathrm{P}$ values below the threshold of .05 were considered significant.

\section{Randomly selecting IGT targets treated with TYP for follow-up WB verification}

To verify the accuracy of the proteomics results, WB was used to randomly verify the expression levels of 16 protein targets involved in the TYP treatment of IGT. For example, the expression levels of Rbp4 (boster, \#PB0368, China) and Flot2 (boster, \#PB0663, China) in the 3 groups were calculated with GAPDH (boster, \#BM1623, China) as an internal reference.

\section{Statistical analysis}

All data in this study were analyzed by SPSS 22.0 software. All results were presented as mean \pm standard error of the mean (SEM). Data were analyzed by one-way ANOVA, with Bonferroni's correction or Student's $t$-test. $\mathrm{P}$ values $<0.05$ were taken to indicate statistical significance.

\section{Results}

\section{Comparison of the body weight and OGTT test results (FBG} and $2 b P G)$ in the 3 groups

As shown in Figure 2A, at the 8th week of intervention, the body weight of the TYP group was lower than that of the IGT model group $(\mathrm{P}<0.05)$. The results indicated that TYP could reduce the weight of IGT rats and improve their obesity status. As shown in Figure 2B,2C, when compared with the IGT model group, FBG in the TYP group showed a downward trend after treatment, but there was no statistical significance $(\mathrm{P}>0.05)$. However, when compared with the IGT model group, $2 \mathrm{hPG}$ in the TYP group decreased $(\mathrm{P}<0.05)$. It can be concluded that TYP can effectively reduce the $2 \mathrm{hPG}$ of IGT in rats.

\section{Observing the morphology of the pancreas by HE staining}

As shown in Figure 2D, the shape of pancreatic islets in the control group was regular, with round cell mass scattered between pancreatic acini. The shape of islet cells was also regular, with a clear boundary and orderly arrangement. Compared with the control group, the islets in the IGT model group had an irregular shape, were atrophied, and had invaded some of the exocrine gland. Furthermore, the size and shape of islet cells were different, the boundary was 

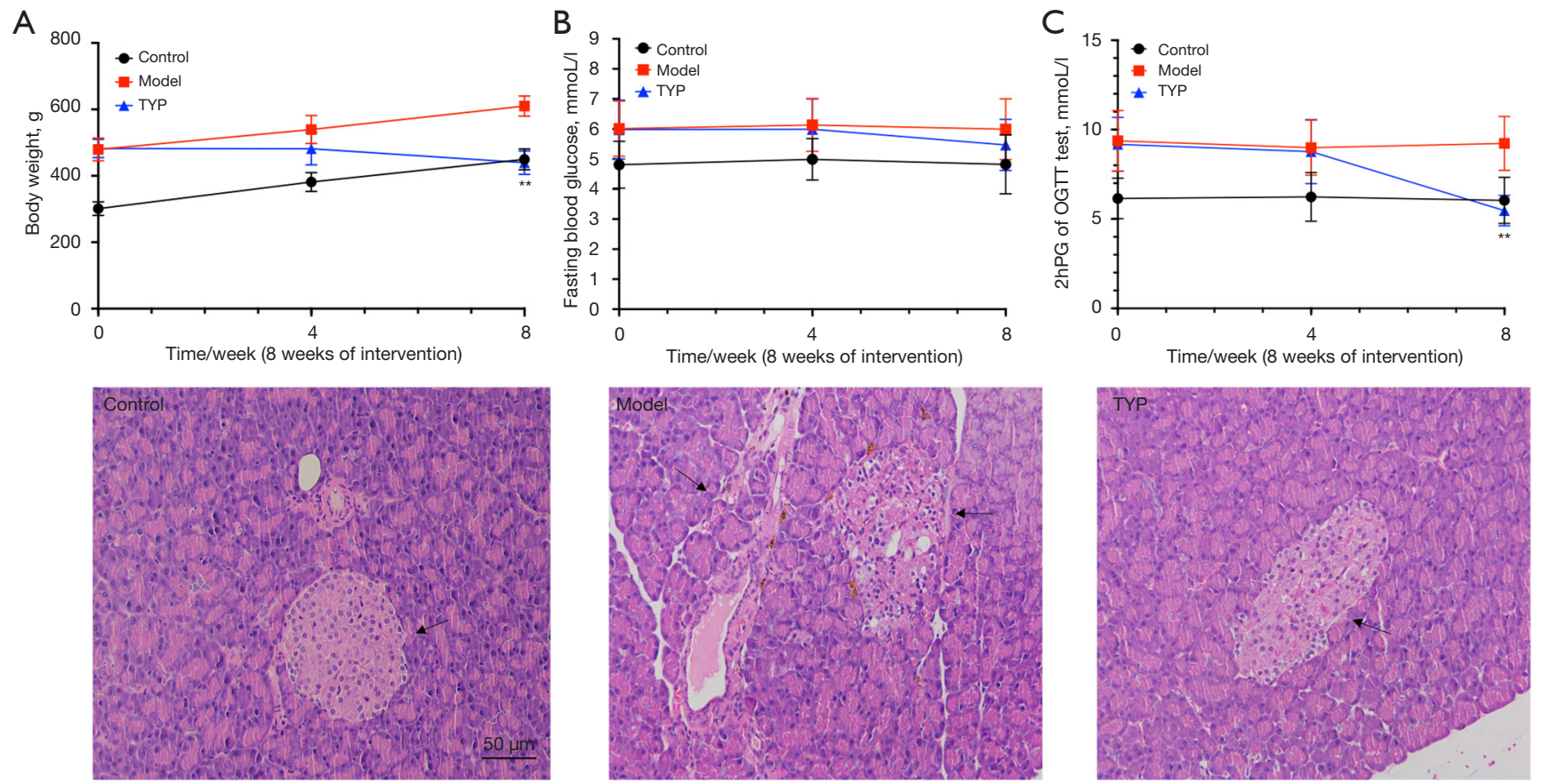

Figure 2 Effects of TYP on weight, blood glucose, and pancreatic morphology after long-term treatment in rats. (A) Weight levels during 8 weeks of administration. (B) FBG levels during 8 weeks of administration. (C) 2 hPG levels from the OGTT test during 8 weeks of administration $(\mathrm{P}<0.05$, vs. IGT). (D) Representative microphotographs of pancreatic sections stained with HE (Scale bar, $50 \mu \mathrm{m}) .{ }^{* *}$, $\mathrm{P}<0.05$, vs. IGT. arrow, islet cells. TYP, Tang-Yi-Ping; FBG, fasting blood glucose; 2hPG, 2-hour plasma glucose; OGTT, oral glucose tolerance test.

unclear, the arrangement was disordered, and the number of islets were reduced. Compared with the IGT model group, the islets in the TYP group were more regular in shape, the boundary was clear, the arrangement was relatively neat, and there were a higher number of islets. This result showed that TYP could improve the morphological damage of the pancreas in rats with IGT, with obvious effects.

\section{Analysis of TMT proteomics results}

\section{Quality control analysis}

For this experiment, we used a Q-Exactive mass spectrometer (Thermo Fisher Scientific Inc.) with highquality precision and high resolution, which could keep good quality deviation in the process of data acquisition, and obtain high-quality MS1 and MS2 spectra. As shown in Figure $3 A$ below, the mass deviation of all identified peptides was mainly within $10 \mathrm{ppm}$, which indicated that the mass deviation of this experiment was low, and the identification results were accurate and reliable. Combined with the strict Mascot analysis tool, the MS data were analyzed to further obtain the score of each MS2. The following Figure 3B shows that the Mascot score of MS2 was ideal, with more than $51.31 \%$ of peptides scoring over 20 points. With a median score of 21 points, it indicated that the quality of the MS experimental data was high. Figure $3 C$ shows that the abundance ratio of most proteins in the (IGT model group $v s$. control group) and (TYP group vs. IGT model group) of equally labeled samples was close to 1 . Overall, the above results showed that good quality deviation was maintained throughout the experiment, and the collected experimental data were reliable.

\section{Identifying DEPs in the 3 groups and the specific} protein targets of TYP to reverse the IGT pathogenesis As shown in Figure 4A, according to the $\mathrm{FC}$ and $\mathrm{P}$ values, there was a total of 104 downregulated DEPs between the IGT model and control groups, and 78 upregulated DEPs between the TYP and IGT model groups. After analyzing the intersection of the DEPs, there were 4 protein targets: Ppif, Slc6a9, Cyp51, and Rab3d. These 4 targets were downregulated after the onset of IGT, and 
A
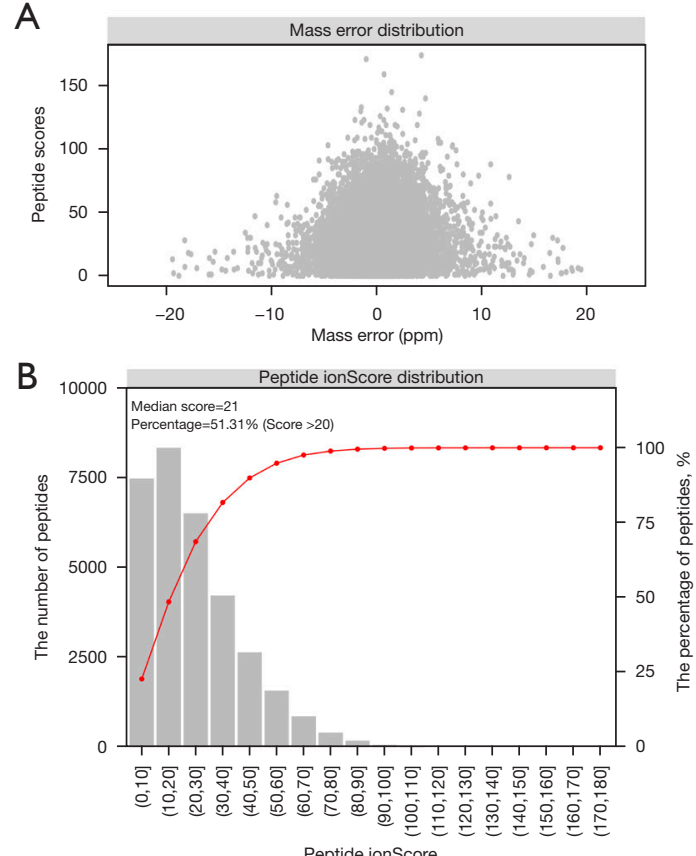
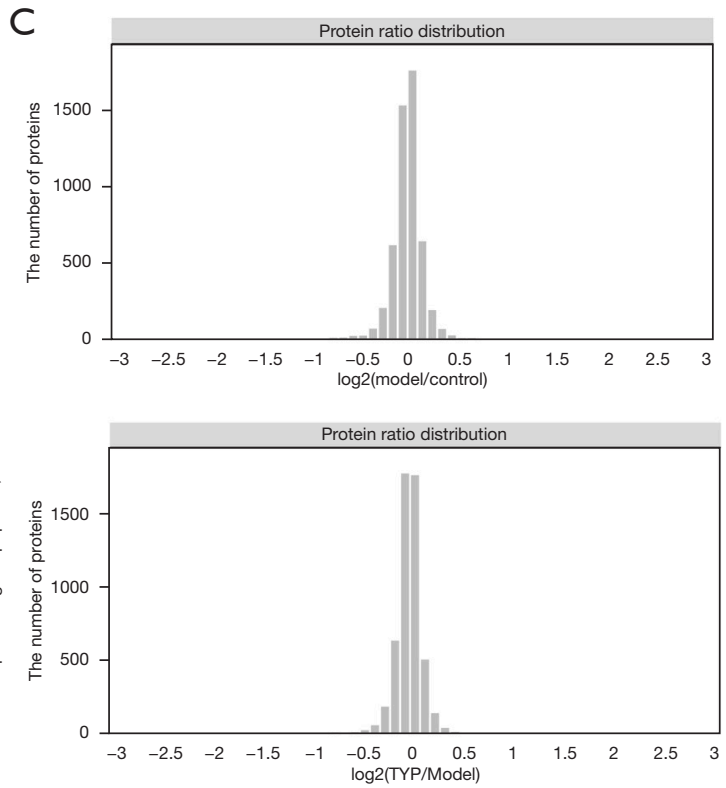

Figure 3 The quality control results of TMT-labeled proteomics. (A) Peptide ion mass deviation distribution. The abscissa is the theoretical mass-to-charge ratio of peptide ions and the mass deviation of the mass-to-charge ratio measured by MS experiments. The unit is ppm, which is one part per million. The ordinate is the Mascot peptide score. (B) Peptide ion score distribution result. (C) The distribution results of protein abundance ratios between groups. The abscissa is FC (logarithmic transformation with base 2), and the ordinate is the number of proteins identified. TMT, tandem mass tag; FC, fold change.

restored to normal expression after the treatment of TYP. Meanwhile, there were 519 upregulated DEPs between the IGT model and control groups, and 59 downregulated DEPs between the TYP and IGT model groups. After analyzing their intersection, we found 12 targets: Man2b1, C7, Tmem181, Chmp4c, Rbp4, Fam3b, Flot2, Macroh2a1, Azgp, Smn1, Ambp, and Mink1. These 12 proteins were upregulated after IGT, downregulated after TYP treatment, and returned to normal expression. We speculated that the 16 proteins obtained from these 2 intersections were the specific therapeutic targets that were abnormal after the onset of IGT, and which tended to be expressed normally after the intervention of TYP. The detailed information of the 16 targets is shown in Table 1. To show the DEPs intuitively between the groups, a volcano map was drawn using the FC and P value ( $t$-test) (Figure $4 B$ ). To classify the DEPs in the groups, a hierarchical cluster algorithm was adopted to analyze the expression patterns of samples between/within groups, to test the rationality of the grouping, and to explain whether the changes in the expression level of differential proteins could represent the significant influence of biological treatment on the samples (Figure 4C). Subcellular organelle is a micro-organ with a certain morphology and function in the cytoplasm (such as mitochondria, endoplasmic reticulum, etc.), which is an important place for proteins to perform different functions (12). Different subcellular organelles often perform different cellular functions, so the analysis of the subcellular localization of proteins helps us to further explore the functions of proteins in cells. The subcellular localization analysis of all DEPs in our study was carried out by using the subcellular structure prediction software Cello. Figure $4 D$ shows the number and distribution ratio of proteins in each subcellular organelle. Of the DEPs in the IGT model and control groups, 165 targets were located in the nucleus, 124 in the cytoplasm, 58 in the plasma membrane, 54 in the extracellular space, 51 in mitochondria, 8 in the lysosomes, and 7 in others (endoplasmic reticulum, etc.). Of the DEPs in the TYP and IGT model groups, 68 proteins were located in the nucleus, 45 in the cytoplasm, 32 in the extracellular space, 25 in the plasma membrane, 15 in the mitochondria, 3 in the lysosome, and 2 in the Golgi 
A

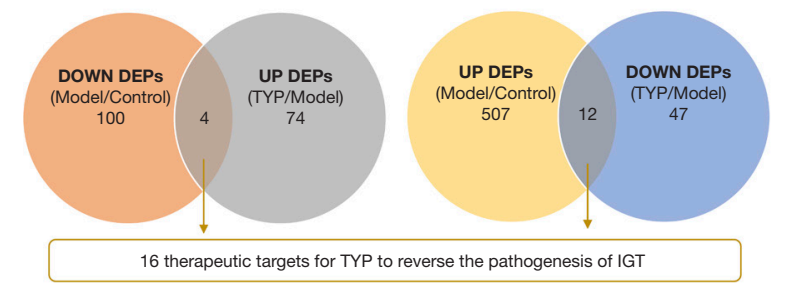

B
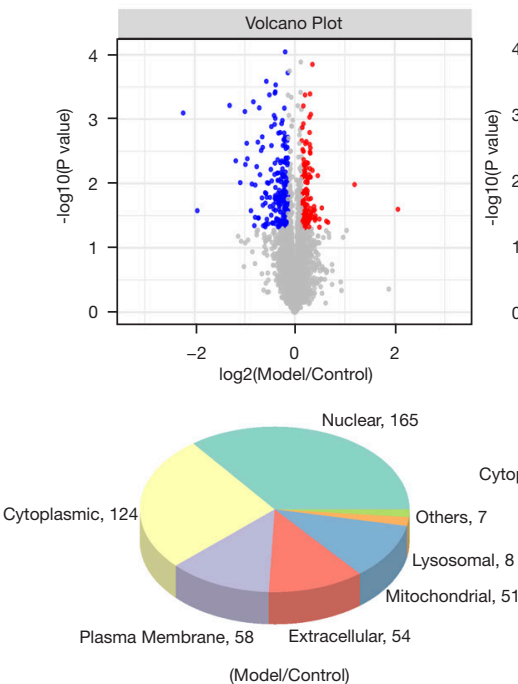

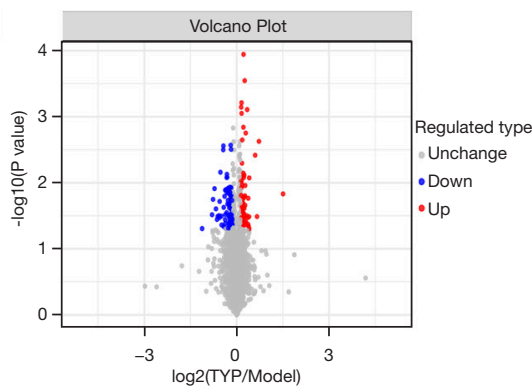

Cytoplasmic, 45
(TYP/Model)

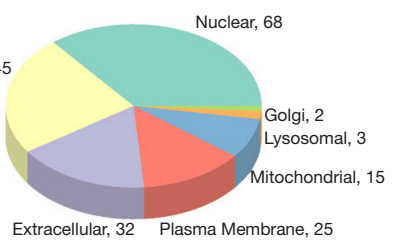

C

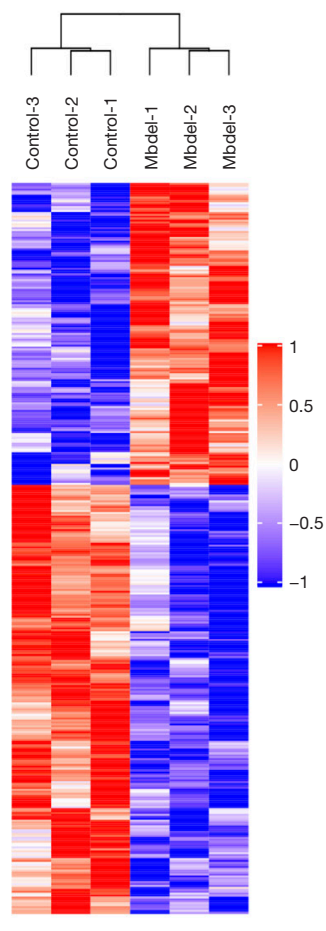

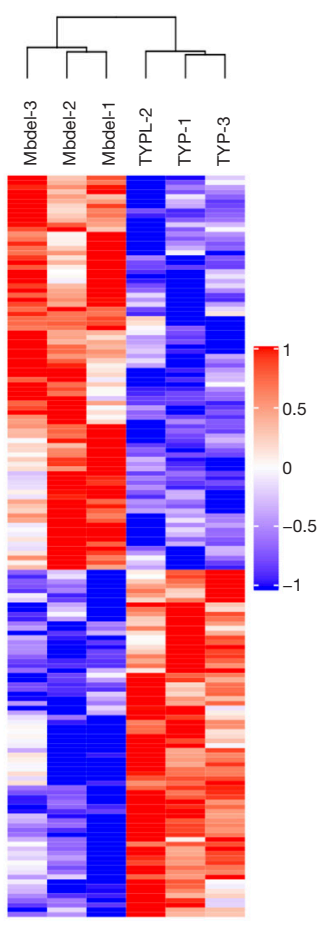

Figure 4 DEPs in the pancreases of the 3 groups. (A) The Venn diagram of up-regulation and down-regulation of DEPs among the 3 groups. (B) The volcano map of DEPs between the groups. The X-coordinate is the differential multiple, and the Y-coordinate is the significant $\mathrm{P}$ value of the difference (logarithmic transformation based on 10). The red dots are the significant DEPs that are upregulated, the blue dots are the downregulated DEPs, and the gray dots are the proteins that have no difference. (C) Hierarchical clustering results. The abscissa is the sample information, and the ordinate is the significantly differentially expressed protein. The expression amount of significantly different proteins in different samples was standardized by $\log 2$ method and displayed in the heat map without color, in which red represents significantly upregulated proteins, and blue represents significantly downregulated proteins. (D) Subcellular localization analysis of proteins differentially expressed between groups.

apparatus.

\section{Biological function analysis}

As shown in Figure $5 A$, the $\mathrm{GO}$ results of the $\mathrm{BP}$ terms (according to $\mathrm{P}<0.05$ ), showed that the $\mathrm{BPs}$ involved in the treatment of IGT mainly included glycerophosphatidylcholine biosynthesis (GO: 0046474), amino acid transport (GO: 0006865), glycerophosphatidylcholine metabolism (GO: 0006650), positive regulation of cell growth (GO: 0030307), regulation of protein stability (GO: 0031647), glycerolipid metabolism (GO: 0046486), cellular amino acid metabolism (GO: 0006520), cellular response to oxidative stress (GO: 0034599), regulation of apoptosis signaling pathway (GO: 2001233), and serine peptidase activity (GO: 0008236), etc.
As shown in Figure 5 B, the results of the KEGG signaling pathway showed that the signaling pathways involved in the TYP treatment of IGT included arginine and proline metabolism, glyceride metabolism, glycerophosphatidylcholine metabolism, mTOR signaling pathway, glycolysis/gluconeogenesis, insulin signaling pathway, and Wnt signaling pathway, etc. $(\mathrm{P}<0.05)$.

\section{Randomly verifying the 16 protein targets through $W B$}

As shown in Figure 6, when compared with the control group, the protein expression levels of Rbp4 and Flot2 in the IGT model group were increased $(\mathrm{P}<0.05)$, and the levels of Rbp4 and Flot2 in the TYP group were decreased $(\mathrm{P}<0.05)$. The expression trend was generally consistent 
Table 1 Specific information on 16 protein targets in the treatment of IGT with TYP

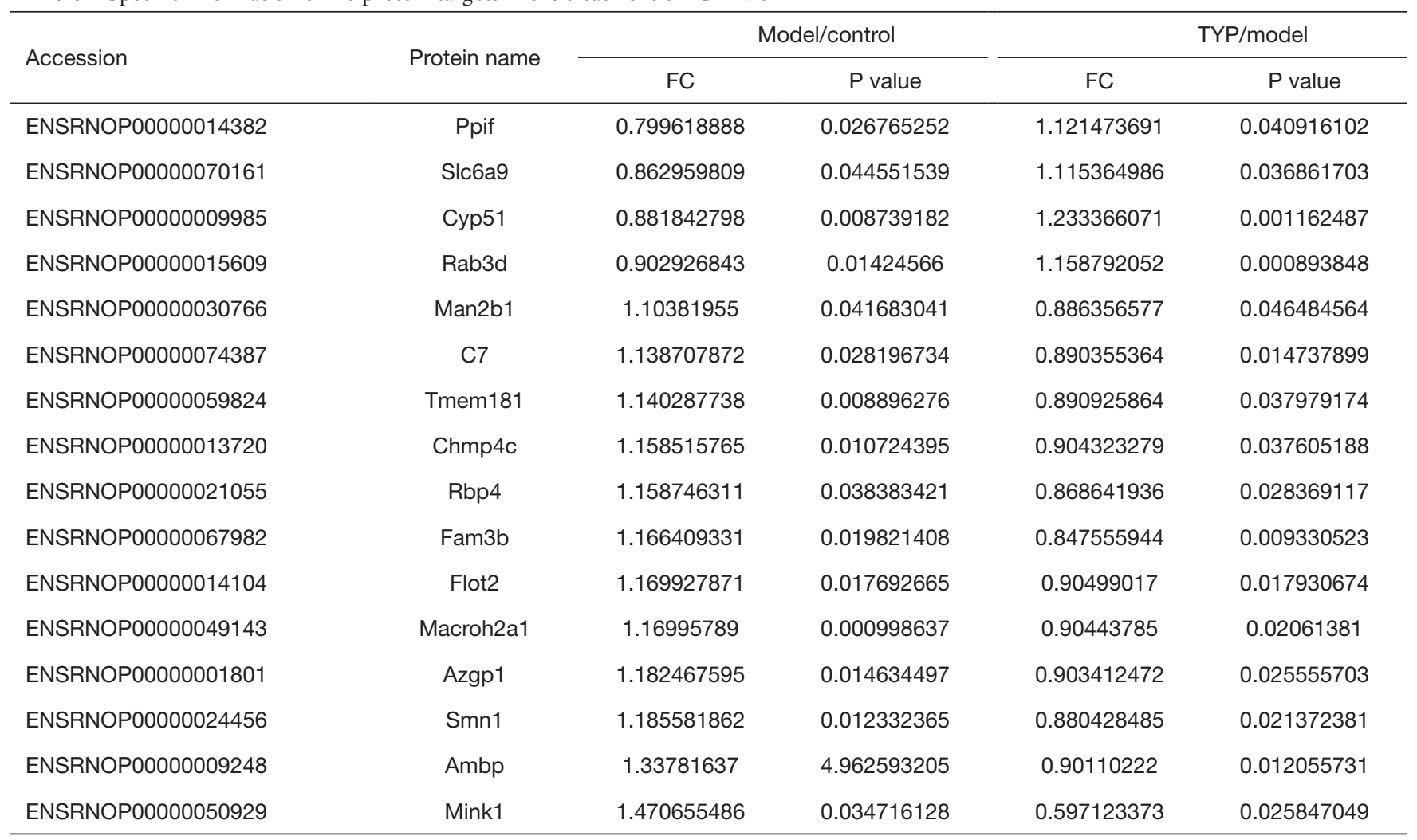

IGT, impaired glucose tolerance; TYP, Tang-Yi-Ping; FC, fold change.

with the results of TMT proteomics, indicating that the result of proteomic analysis was reliable in this study.

\section{Discussion}

IGT-related postprandial hyperglycemia in the prediabetic stage is associated with the onset and incidence of T2DM (13). As the IGT stage is the only stage that DM can be reversed and controlled, its prevention and treatment is of great significance. The main pathological characteristics of IGT are decreased glucose utilization and tolerance, insulin resistance, and decreased function of islet $\beta$ cells, resulting in relative or absolute insufficiency of insulin secretion and higher postpranpranal blood glucose level. In addition, the pathophysiological changes in IGT are manifested as both glucose metabolism disorders, and abnormal protein and fat metabolism, which are manifested as obesity, increased triglyceride level, decreased high-density lipoprotein cholesterol (HDC), increased low-density lipoprotein cholesterol (LDL-C), hyperuricemia, and hyper fibrinogen (4). The treatment of
IGT includes both lifestyle and drug interventions. The fundamental lifestyle intervention has to do with weight loss in individuals with obesity or overweight, and the key to weight loss is rational diet and moderate exercise. Improving lifestyle is economical and effective, but many IGT patients find it difficult to adhere to a strict diet program for a long time. Therefore, drugs are also a useful source of intervention in IGT. Currently, there are very few drugs used in the treatment of IGT, the main ones being metformin and acarbose. However, these treatments have certain limitations due to some adverse side effects, such as hepatorenal toxicity, gastrointestinal reactions, etc. A series of studies have confirmed that TYP can invigorate the spleen, relieve the depressed liver, treat IGT effectively and safely, improve the proliferation of pancreatic $\beta$-cells, and protect the number and function of $\beta$-cells. Based on previous research, TYP's mechanism relates to the downregulation of TXNIP and the promotion of the expression of MaFA $(8,9)$.

The results of our study show that TYP has a comprehensive effect on the regulation of weight loss and 

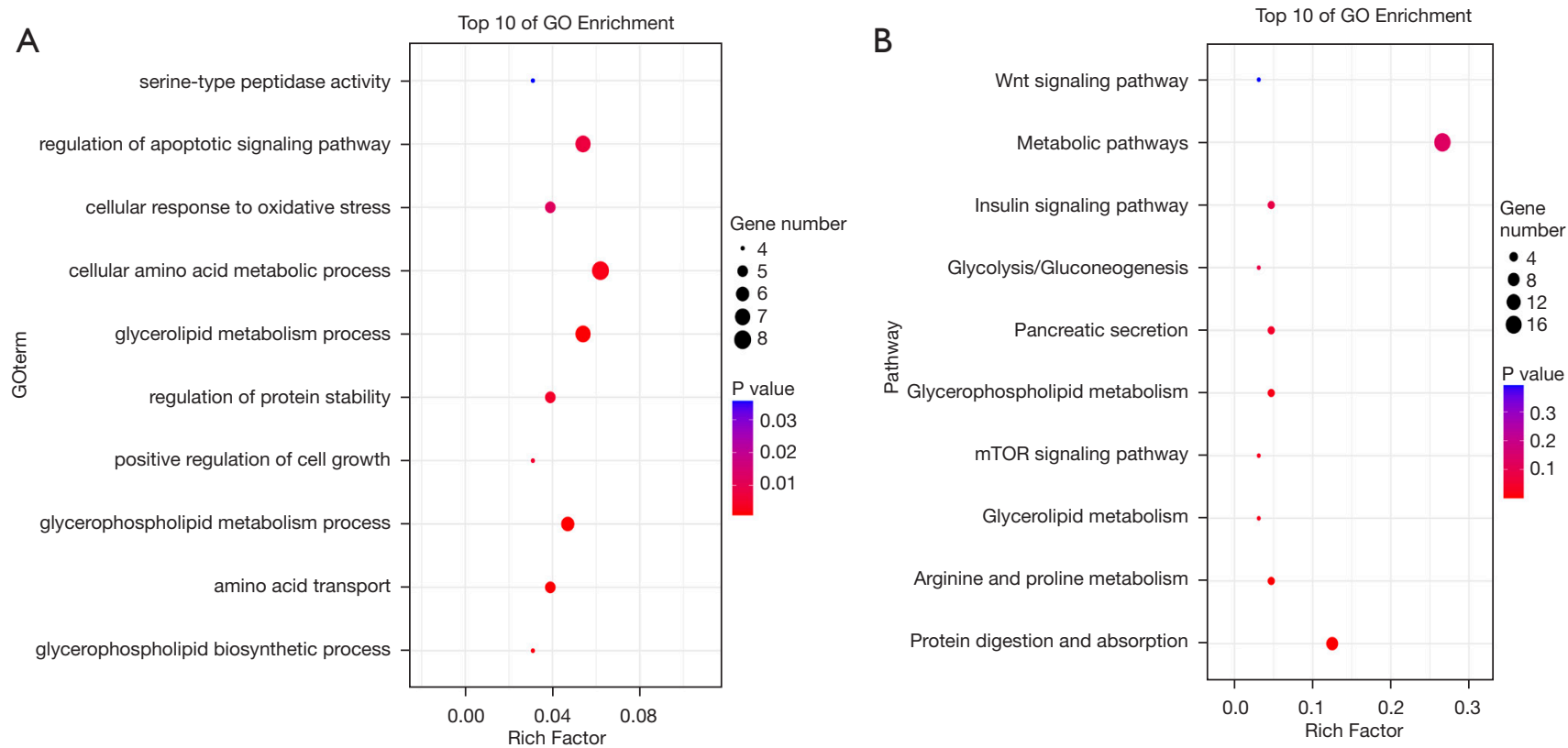

Figure 5 Biological function enrichment analysis results of DEPs between the TYP group and the IGT model group. (A) GO enrichment function analysis result. The abscissa is the rich factor, and the ordinate is the name of the GO term (BP). (B) KEGG enrichment function analysis result. The abscissa represents the rich factor, and the ordinate represents the signaling path name. GO, Gene Ontology; KEGG, Kyoto Encyclopedia of Genes and Genomes.
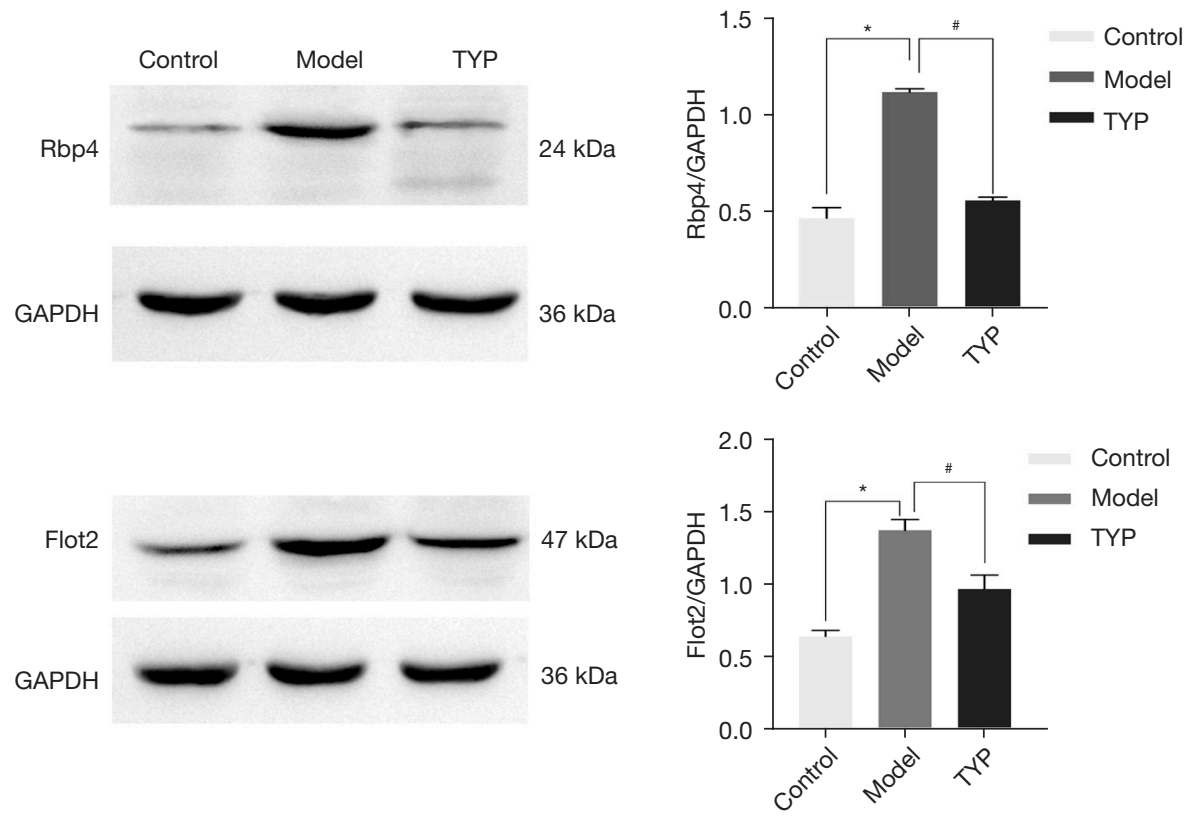

Figure 6 Relative expression levels of Rbp4 and Flot2 proteins in pancreatic samples of the 3 groups. Rbp4: *, $\mathrm{P}<0.05, v s$. Control; ${ }^{*}, \mathrm{P}<0.05$, vs. IGT. Flot: *, $\mathrm{P}<0.05$, vs. Control; \#, $\mathrm{P}<0.05$, vs. IGT. IGT, impaired glucose tolerance. 
hypoglycemia in rats with IGT, and that it can improve the morphology of rat islets with a definite curative effect. The TMT technique, developed by Thermo Scientific in the US, is one of the most commonly used high-throughput screening techniques in quantitative proteomics. TMT proteomics can identify and analyze changes in proteins from a holistic perspective and can identify the occurrence and development of diseases and all targets of drug action by monitoring abnormal changes in the expression of the protein. Currently, the application of this technology to explore the mechanism of drug treatment in endocrine and metabolic diseases including DM has become a major focus in the medical field. Therefore, through TMT proteomics in this study, we were able to identify the specific target proteins, BPs, and main signaling pathways of TYP in IGT rats. This indicates that TYP has the characteristics of multiple targets and pathways in the treatment of IGT. In light of the results of TMT proteomics, we found many DEPs in the 3 groups. This shows that, to a certain extent, TYP regulates the differential expression of 16 protein targets (PPif, Slc6a9, Cyp51, Rab3d, Man2b1, C7, Tmem181, Chmp4c, Rbp4, Fam3b, Flot2, Macroh2a1, Azgp1, Smn1, Ambp, and Mink1) in the rat pancreas. From our findings we have concluded that these 16 proteins are the specific therapeutic targets that are abnormal after IGT onset, and which tend to return to normal after TYP intervention.

Several targets have been reported to be closely related to the occurrence of DM, such as the retinol binding protein 4 (Rbp4). A member of the retinol-binding protein (RBP) family, Rbp4 is a newly discovered adipose-derived circulating factor which is mainly expressed and secreted in adipose tissue, can increase the susceptibility of DM, and is related to obesity, IR, and insulin secretion (14). Another target, Fam3b (PANDER), is a cytokine that is specifically highly expressed in pancreatic $\beta$-cells and is located in the same secretion granule as insulin. Overexpression of Fam $3 \mathrm{~b}$ in islets leads to mass apoptosis of islet $\beta$-cells. Previous experiments have confirmed that high-doses of Fam $3 \mathrm{~b}$ can induce apoptosis in pancreatic $\beta$-cells and inhibit both insulin secretion and insulin signal transduction in hepatocytes. This suggests that Fam $3 b$ may have a certain relationship with the occurrence and development of T2DM (15). A previous study (16) has shown that the overexpression of Fam $3 \mathrm{~b}$ in the liver of C57 mice could quickly cause significant liver enlargement, fatty liver, hyperlipidemia, IR, and other related metabolic changes. These effects oppose that of Fam3a, which can improve metabolic status. Therefore, activating Fam3a and/or inhibiting Fam3b from correcting the unbalanced regulatory network between them is expected to become a new strategy for the prevention and treatment of DM (17). Zhang (18) found that Fam3b mediated the proliferation and migration of VSMCs induced by high glucose through inhibiting miR-322-5p. He also speculated that Fam3b might become a new target for the treatment of DM-related cardiovascular diseases. Furthermore, complement 7 (C7) is closely related to Early Diabetic Nephropathy (EDN), and may serve as a biomarker and molecular therapeutic target for the detection and treatment of EDN (19). Some of the other targets are closely related to lipid metabolism, such as Azgp1 and Flot2. Azgp1 (zinc $\alpha 2$ glycoprotein) is a new type of adipocytokine factor that exists in human plasma and a variety of body fluids. Azgp1 is not only involved in the regulation of obesity and lipid metabolism but is also closely related to T2DM. Their mechanism may be related to increasing the tissue glucose metabolism rate, and through binding to $\beta 3$ adrenergic receptors (20). Flot2 is also a new type of adipocyte factor, which is closely related to the occurrence of high triglycerides, obesity, and DM. This study verified the protein expression level of Flot2 through $\mathrm{WB}$, and the results showed that the expression level of Flot2 in the IGT model group increased and decreased. The expression trend was consistent with the TMT proteomics results. According to the results of GO analysis, the BPs involved in TYP included a variety of lipid metabolic processes, such as glycerophospholipid biosynthesis (GO: 0046474), glycerophospholipid metabolism (GO: 0006650), glycerolipid metabolism (GO: 0046486), and so on. It is also worth noting that among the 16 protein targets identified as working against IGT after TYP, some of them, such as Ambp and Mink1, have not previously been reported to be closely related to the pathogenesis and treatment of DM. From this we speculate that these targets are expected to become new targets for DM, and will lead to new strategies for the treatment of both IGT and DM. This is something we will further verify in follow-up experiments.

The results of the KEGG analysis indicated that the signaling pathways involved in the treatment of IGT with TYP mainly included arginine and proline metabolism, glyceride metabolism, glycerophospholipid metabolism, mTOR signaling pathway, glycolysis/gluconeogenesis, insulin signaling pathway, and Wnt signaling pathway, etc.

The metabolic pathways of arginine and proline are closely related to DM and other complications. The GO enrichment analysis results of this experiment also 
showed that the BPs involved in TYP treatment included amino acid transport (GO:0006865), cellular amino acid metabolism (GO:0006520), and so on. Previous studies have confirmed that a herb called BerberiskansuensisSchneid could improve the pathological changes and pharmacodynamic indicators of DM. The herb's mechanism might relate to an increase in the level of ornithine in the serum of DM rats, and through its ability to aid the metabolism of arginine and proline (21). This study confirmed that TYP may reduce blood glucose and body weight by regulating arginine and proline metabolism. Another signaling pathway, The Wnt, is closely related to the regeneration of islet cells, hepatic glucose and lipid metabolism, and IR. The inhibition of the Wnt signaling pathway has been noted to be a risk factor for T2DM (22). Furthermore, experiments by Dabern have shown that abnormalities in the Wnt/ $\beta$-catenin signaling pathway may cause disorders in glucose metabolism, increase the risk of DM, and participate in the transdifferentiation process of renal epithelial cells, which are mediated by high glucose (23). The mTOR is a serine-/threonine-protein kinase with a highly conserved structure and function, which mainly exists in vivo as a complex of mTORCl and mTORC2 (24). As a nutrient sensor, mTORC1 is located at the center of a complex signaling network and is a regulatory protein for a variety of key signaling pathways in cells. mTORC1 signaling is highly related to pancreatic islet cell function and activating mTORC1 signaling in pancreatic islet epithelial cells leads to upregulation of insulin levels and glucose stimulating insulin (GSIS) (25). Yin (26) observed the therapeutic effects of ShenzhuTiaopi Granules in DM rats and found that they could invigorate the spleen, reduce IR, and eliminate dampness and resolve phlegm. Yin's results showed that ShenzhuTiaopi Granules could significantly reduce FPG levels in Goto-Kakizaki (GK) rats, improve liver fat deposition, and IR. The mechanism of these granules was related to the regulation of the AMPK/mTOR pathway. This study confirmed that TYP can play a hypoglycemic role in the treatment of IGT by regulating the mTOR signaling pathway. All in all, in this study TYP can be seen to have the characteristics of multiple targets and pathways in the treatment of IGT.

Despite our findings, there are still some shortcomings evident in our experiment. For example, due to time and funding limitations, only 3 pancreatic samples were selected for analysis in each group, and the 16 protein targets screened in this experiment were only partially verified by western blot. In our follow-up study, we will expand the scope of protein target verification.

\section{Conclusions}

This study is the first report to use TMT-labelled quantitative proteomics technology and bioinformatics to identify the protein targets, BPs, and related signaling pathways involved in the TYP treatment of IGT in rats. TYP regulates 16 protein targets and further regulates multiple biological functions and multiple signal transduction pathways for anti-IGT therapy. Our study provides a new approach for finding potential therapeutic targets to improve the treatment of IGT.

\section{Acknowledgments}

Funding: This study was supported by the National Natural Science Foundation of China (No: 81673966) and Shandong Province' Taishan Scholar Project Special Funding (No: ts201712097).

\section{Footnote}

Reporting Checklist: The authors have completed the ARRIVE reporting checklist. Available at https://dx.doi. org/10.21037/atm-21-4257

Data Sharing Statement: Available at https://dx.doi. org/10.21037/atm-21-4257

Conflicts of Interest: All authors have completed the ICMJE uniform disclosure form (available at https://dx.doi. org/10.21037/atm-21-4257). The authors have no conflicts of interest to declare.

Ethical Statement: The authors are accountable for all aspects of the work in ensuring that questions related to the accuracy or integrity of any part of the work are appropriately investigated and resolved. All animal experiments protocol were performed strictly in compliance with Chinese guidelines, including the Standards for Laboratory Animals (GB14925-2001) and the Guideline on the Humane Treatment of Laboratory Animals (MOST 2006a). All animal procedures were approved by the Animal Ethics Committee of Shandong University of Traditional Chinese Medicine (No. SDUTCM20190520001).

Open Access Statement: This is an Open Access article 
distributed in accordance with the Creative Commons Attribution-NonCommercial-NoDerivs 4.0 International License (CC BY-NC-ND 4.0), which permits the noncommercial replication and distribution of the article with the strict proviso that no changes or edits are made and the original work is properly cited (including links to both the formal publication through the relevant DOI and the license). See: https://creativecommons.org/licenses/by-nc-nd/4.0/.

\section{References}

1. Bansal N. Prediabetes diagnosis and treatment: A review. World J Diabetes 2015;6:296-303.

2. Saeedi P, Petersohn I, Salpea P, et al. Global and regional diabetes prevalence estimates for 2019 and projections for 2030 and 2045: Results from the International Diabetes Federation Diabetes Atlas, 9th edition. Diabetes Res Clin Pract 2019;157:107843.

3. Yip WCY, Sequeira IR, Plank LD, et al. Prevalence of PreDiabetes across Ethnicities: A Review of Impaired Fasting Glucose (IFG) and Impaired Glucose Tolerance (IGT) for Classification of Dysglycaemia. Nutrients 2017;9:1273.

4. Tabák AG, Herder C, Rathmann W, et al. Prediabetes: a high-risk state for diabetes development. Lancet 2012;379:2279-90.

5. Aroda VR, Knowler WC, Crandall JP, et al. Metformin for diabetes prevention: insights gained from the Diabetes Prevention Program/Diabetes Prevention Program Outcomes Study. Diabetologia 2017;60:1601-11.

6. Warrilow A, Somerset S, Pumpa K, et al. Metformin use in prediabetes: is earlier intervention better? Acta Diabetol 2020;57:1359-66.

7. Moelands SV, Lucassen PL, Akkermans RP, et al. Alphaglucosidase inhibitors for prevention or delay of type 2 diabetes mellitus and its associated complications in people at increased risk of developing type 2 diabetes mellitus. Cochrane Database Syst Rev 2018;12:CD005061.

8. Huang YQ, Yang QF, Wang H, et al. Long-term clinical effect of Tangyiping Granules () on patients with impaired glucose tolerance. Chin J Integr Med 2016;22:653-9.

9. Huang J. Tangyiping improves glucose tolerance and reduces islets through TXNIP/miR-204/MafA signaling pathway $\beta$ Experimental study on cell function and clinical efficacy evaluation. Shandong University of Traditional Chinese Medicine, 2019.

10. Kanat M, DeFronzo RA, Abdul-Ghani MA. Treatment of prediabetes. World J Diabetes 2015;6:1207-22.

11. Bartoli E, Fra GP, Carnevale Schianca GP. The oral glucose tolerance test (OGTT) revisited. Eur J Intern Med 2011;22:8-12.

12. Sasaki K, Yoshida H. Organelle Zones. Cell Struct Funct 2019;44:85-94.

13. Daniele G, Abdul-Ghani M, DeFronzo RA. What are the pharmacotherapy options for treating prediabetes? Expert Opin Pharmacother 2014;15:2003-18.

14. Huang R, Bai X, Li X, et al. Retinol-Binding Protein 4 Activates STRA6, Provoking Pancreatic $\beta$-Cell Dysfunction in Type 2 Diabetes. Diabetes 2021;70:449-63.

15. Zhang X, Yang W, Wang J, et al. FAM3 gene family: A promising therapeutical target for NAFLD and type 2 diabetes. Metabolism 2018;81:71-82.

16. Moak SL, Dougan GC, MarElia CB, et al. Enhanced glucose tolerance in pancreatic-derived factor (PANDER) knockout C57BL/6 mice. Dis Model Mech 2014;7:1307-15.

17. Wilson CG, Robert-Cooperman CE, Burkhardt BR. PANcreatic-DERived factor: novel hormone PANDERing to glucose regulation. FEBS Lett 2011;585:2137-43.

18. Zhang $W$, Chen S, Zhang Z, et al. FAM3B mediates high glucose-induced vascular smooth muscle cell proliferation and migration via inhibition of miR-322-5p. Sci Rep 2017;7:2298.

19. Sircar M, Rosales IA, Selig MK, et al. Complement 7 Is Up-Regulated in Human Early Diabetic Kidney Disease. Am J Pathol 2018;188:2147-54.

20. Pearsey HM, Henson J, Sargeant JA, et al. Zinc-alpha2glycoprotein, dysglycaemia and insulin resistance: a systematic review and meta-analysis. Rev Endocr Metab Disord 2020;21:569-75.

21. Ai XP, Wang XB, Wang XY, et al. The protective mechanism of Berberis on STZ induced diabetic nephropathy in rats based on metabonomics. Chinese Pharmacology and Clinic 2019;35:67-73.

22. Xi XH, Wang FW, Wang Y, et al. Alteration of Wnt/ $\beta$-catenin signaling pathway in early diabetic rats'myocardium. Chin Pharmacol Bull 2015;31:363-6.

23. Yan Z, Yao F, Zhang LP, et al. Modulation of Wnt/ $\beta$-catenin signaling pathway byirbesartan in high glucose induced tubular epithelial mesenchymal transition. Chin Pharmacol Bull 2009;25:1630-4.

24. Saxton RA, Sabatini DM. mTOR Signaling in Growth, Metabolism, and Disease. Cell 2017;168:960-76.

25. Mao Z, Zhang W. Role of mTOR in Glucose and Lipid Metabolism. Int J Mol Sci 2018;19:2043.

26. Yin Y, Fang Z, Wu Y, et al. Effect of Shenzhu Tiaopi 
granule on hepatic insulin resistance in diabetic Goto-Kakizakirats via liver kinase B1/adenosine 5'-monophosphate/mammalian target of rapamycin signaling pathway. J Tradit Chin Med 2021;41:107-16.

(English Language Editor: J. Goetz)

Cite this article as: $\mathrm{Li} \mathrm{J,} \mathrm{Bu} \mathrm{S,} \mathrm{Zhou} \mathrm{H,} \mathrm{Bi} \mathrm{S,} \mathrm{Xu} \mathrm{Y.}$ Identifying potential therapeutic targets of Tang-Yi-Ping for the treatment of impaired glucose tolerance: a tandem mass tag-labeled quantitative proteomic analysis. Ann Transl Med 2021;9(20):1532. doi: 10.21037/atm-21-4257 